\title{
A WEB-BASED GIS PORTAL FOR SIMULATING GEODETIC CONTROL NETWORKS IN REPUBLIC OF KENYA
}

\author{
David Maina NDIRANGU(i)*, Benson Kipkemboi KENDUIYWO (D), \\ Edward Hunja WAITHAKA (D) \\ Department of Geomatic Engineering and Geospatial Information Systems (GEGIS), \\ Jomo Kenyatta University of Agriculture and Technology, Nairobi, Kenya
}

Received 13 December 2019; accepted 05 December 2020

\begin{abstract}
Geodetic networks development begun in Kenya and Africa as a whole at the dawn of 20th century. Consequently, enormous geodetic data has been realized. In Kenya, the data was recorded in form of paper trigonometric cards, paper topographic maps, and paper cadastral map sheets and centrally archived in the ministry of lands and physical planning headquarters in Nairobi. This was to assist locate and visualize suitable survey of Kenya geodetic pillar of interest to user. However, the user still has to commute to the headquarters in order to physically acquire coordinate information of any pillar in the country. This circumstance has fabricated a framework that has triggered accumulation of millions of paper records. The effectiveness and efficiency of serving the users is greatly undermined by the manual process. Therefore, an alternative solution is necessary to alleviate dependence on an outdated manual process. As a result, this study sought to fill this gap by designing a web geoportal for management of geodetic control networks and user access which incorporates making of payments of coordinates in different systems remotely. The geoportal comprises of an integration of a database management system, a server configuration and a website with an automated data access through a payment gateway. Java scripts and python programming languages were used. The final platform has the following capabilities: spatial visualization, co-ordinates system conversion, online payment, and request and access of data remotely. We foresee that the system will aid the ministry of lands and physical planning to disseminate geodetic information to users efficiently and effectively while tracking revenue payments.
\end{abstract}

Keywords: geodetic control network, web GIS, geoportal, payment gateway.

\section{Introduction}

In Kenya, the lead agency for provision of geodetic data services is survey of Kenya (Owino, 2013). The geodetic network major triangulation was based on Cassini coordinate system (Lwangasi, 1993; Mugnier, 2014). It was established to fill the void of the need of registration of title survey and to provide controls for topographical mapping. This geodetic network extension continued from 1906 to 1914 and from 1939 to 1941 (Lwangasi, 1993). Further, in Sir David Gill (Stone, 1976) together with two British astronomers established a different datum and a triangulation network in 1897. Sir Thomas Maclear had preceded Sir David Gill in 1837 to measure arc of meridian, a one degree of longitude starting from cape town table mountains to Piketburg (Aurora) in south Africa (Rainsford, 1951). Where, his work was to verify and extend arc of meridian previously measured by French Abbe
N.L. De la Caille some 80 years earlier (Wood, 2014) as well provide geodetic control for topographic maps and navigation charts (Rainsford, 1951; Stone, 1976; Wood, 2014). Besides, measurement and establishment of a second arc of meridian (30th arc of meridian) in 1897 (Wood, 2014), the triangulation network was later extended to cover the whole of South Africa by Sir David Gill (Wood, 2014). Further extension of the triangulation network at the 30 degrees east of Greenwich prime meridian, from South Africa to East Africa was completed in 1950 (Lwangasi, 1993).

On the line of the advancement of technology, the decision was made on establishment of Kenya National Networks based on global navigation and satellite system (GNSS) technology and with global connection (continuous operating reference system) by lead mapping agency in 2009 (Mugnier, 2014; Murage et al., 2008). This decision

*Corresponding author. E-mail: davidmaina333@gmail.com

Copyright $\odot 2020$ The Author(s). Published by Vilnius Gediminas Technical University

This is an Open Access article distributed under the terms of the Creative Commons Attribution License (http://creativecommons.org/licenses/by/4.0/), which permits unrestricted use, distribution, and reproduction in any medium, provided the original author and source are credited. 
was arrived at as a result of inefficiency of existing triangulation networks, which are based on old technologies, most monuments are destroyed, not consistent, heights not accurate, located on hill tops, not safe, and not easy to access (Murage et al., 2008). As a result of the diverse types of geodetic control networks vast amount of data has been accumulated. This geodetic data also should be delivered to the public for use by various entities in the country. However, the challenge that is detrimental to the provision of services by various government institutions is the paper based mode of services provision and data exchange (Mabeya \& Waithaka, 2014; Magondu et al., 2012; Mwaura \& Waithaka, 2013; Wanjohi \& Mutua, 2015). Geodetic control network data is also not an exception. The data storage, data retrieval and data publication is manual and paper based. For instance, geodetic data is paper based, stored in form of trigonometric index cards and brochure or leaflets, archived in shelfs. This data is centrally located at survey of Kenya headquarter offices in Nairobi and is intended to serve the whole country. Retrieval of such hard copy data generated by different departments is ineffective, time consuming, un-reliable, and inefficient. This has led to uneconomical productivity in service delivery (Wanjohi \& Mutua, 2015).

Therefore, this study seeks to design and implement a geodetic network geo-portal which would enable survey of Kenya staff to document and update the data. We also integrate a system for visualization of controls, conversion of coordinates between different systems, request for access and payment of coordinates remotely. The payment gateway, web processing and conversion of coordinates online is an additional feature that previous studies had not intervened.

\section{Materials and methods}

\subsection{Study area}

Kenya is one of the African countries situated on the Equator in eastern Africa region. It borders Ethiopia on North-East, South Sudan on South West, Indian Ocean on South East and Uganda on the West (Figure 1). Kenya has diverse topographic features; Great Rift Valley, highlands, lakes, plateaus, arid and semi-arid landscapes, volcanic landforms are amongst wide range of topographical features located in Kenya. Most of trigonometric geodetic network pillars in Kenya are located on the hills.

The trigonometric stations are categorized into first, second, third and fourth order. First and second order trigonometric stations were used in this study giving a total number of 500 geodetic pillars. In recent years the GNSS CORS are being installed to replace trigonometric stations. 26 GNSS CORS stations were used in the study.

\subsection{Data description}

We collected data as per users' needs assessment as illustrated by summary in Table 1 . This comprised of various maps which facilitated preparation of spatial catalogue data such as the shape files of Kenya, water bodies and towns. Resultant data was therefore used to set up a base map and the entire geodatabase for the geodetic geoportal.

Quantum GIS, open source software was used in data processing and most importantly in preparation of georeferenced thematic layers, assigning of geographic coordinates for spatial reference and development of digital maps. These resulted into spatially referenced geographic data with both cartographic and locational aspects.

Further, trigonometric index card in the Survey of Kenya archives were scanned and digitized into vector

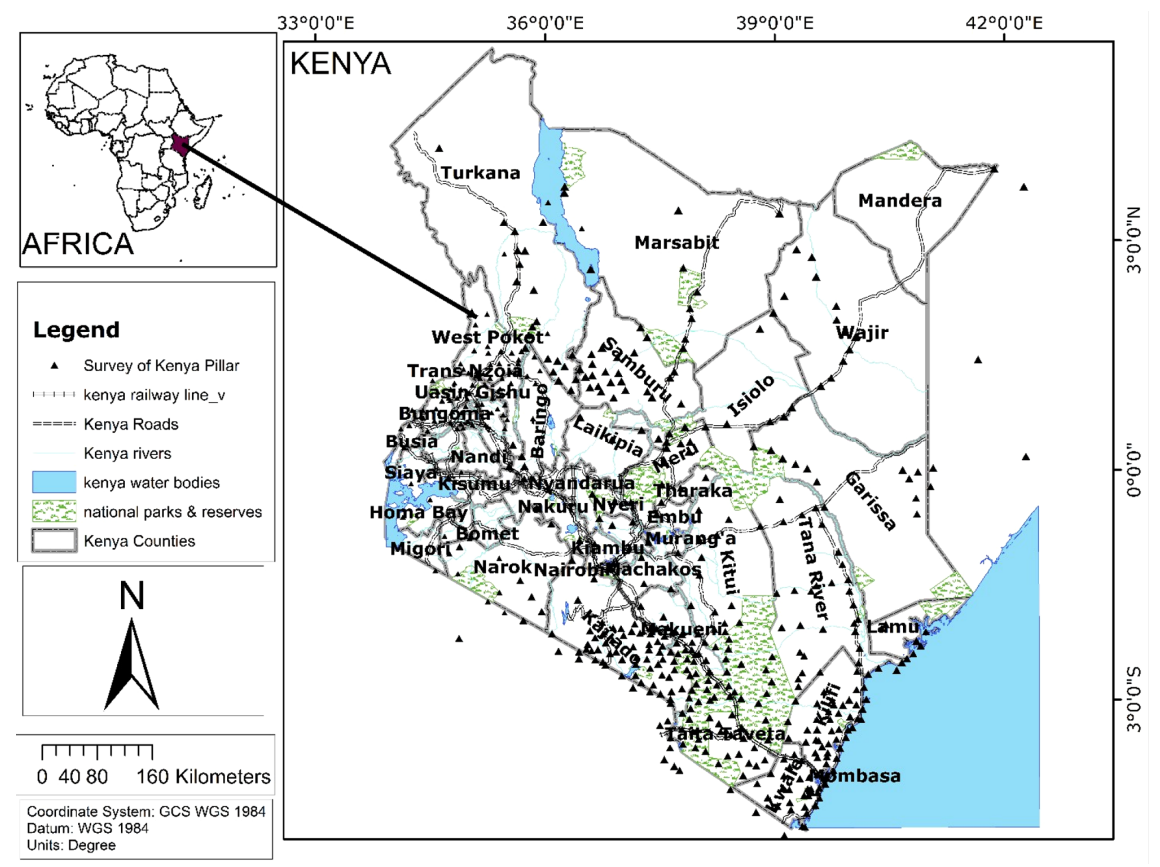

Figure 1. Study area locational map 
formats in form of shape files. Spatial referencing was done to align data frames to actual geographic coordinates on the surface of the earth.

Table 1. Data type format and source

\begin{tabular}{|l|l|l|l|}
\hline \multicolumn{1}{|c|}{ Data } & \multicolumn{1}{|c|}{$\begin{array}{l}\text { Class of } \\
\text { Beacons }\end{array}$} & \multicolumn{1}{|c|}{ Format } & \multicolumn{1}{|c|}{ Source } \\
\hline $\begin{array}{l}\text { Kenya, water } \\
\text { bodies, towns }\end{array}$ & primary & $\begin{array}{l}\text { ESRI shape } \\
\text { file }\end{array}$ & Survey of Kenya \\
\hline Africa & primary & $\begin{array}{l}\text { ESRI shape } \\
\text { files }\end{array}$ & Survey of Kenya \\
\hline $\begin{array}{l}\text { Geodetic pillar } \\
\text { terrestrial }\end{array}$ & primary & $\begin{array}{l}\text { Hard copy } \\
\text { cards }\end{array}$ & Survey of Kenya \\
\hline $\begin{array}{l}\text { Geodetic pillar } \\
\text { (continuous } \\
\text { operating } \\
\text { system) }\end{array}$ & primary & $\begin{array}{l}\text { Hard copy } \\
\text { cards }\end{array}$ & Survey of Kenya \\
\hline $\begin{array}{l}\text { AFREF pillar } \\
\text { (continuous } \\
\text { operating } \\
\text { system) }\end{array}$ & primary & $\begin{array}{l}\text { Hard copy } \\
\text { cards }\end{array}$ & $\begin{array}{l}\text { Regional Centre } \\
\text { of Mapping of } \\
\text { Resources for } \\
\text { Development }\end{array}$ \\
\hline
\end{tabular}

\subsection{Methods}

The main aim of this research was to design a web geoportal for management of geodetic control networks, dynamic visualizations and coordinate information access via a payment gateway in Kenya. To achieve the mentioned objective, we adopted the methodological approach shown in Figure 2. The geoportal comprises of an integration of a database management system, a server configuration and a website with a coordinate information access payment gateway tool. To implement these applications, we used Apache Tomcat, PostgreSQL as the database, GeoServer, Apache Directory Server and java script, and python programming languages for application of front end and back end.

Entry of non-spatial data section into the database (Appendix 3) was accomplished for explicit layers utilizing the attributes data captured. Therefore, all coordinates in universal transverse Mercator were input in the data base, datum, ellipsoid, destroyed points or not destroyed tuples were generated and also data on re-establishment of points was done for the cases where they are initially destroyed.

The process was done repeatedly for all spatial catalogue layers that were generated and the output was a map for visualization and which was to be tiled in the spatial catalogue of the application, in the research study area; that was utilized in the later procedures in the implementation of the framework portal. After a conclusive process of map creation, from the zone of interest, layers which subsequently needed development of a database were exported into the PostGIS database, the spatial component of PostgreSQL. These different layers were linked with the PostgreSQL database and then added to
Quantum GIS as PostgGIS layers. Finally, by utilizing the Quantum GIS capacity and capability to export data, a Map file was generated which was then utilized in the improvement of the framework utilizing the Geonode application.

\subsubsection{Database design}

We used PostgreSQL and PostGIS database server to design a database for spatial and non-spatial data respectively. PostgreSQL database management system was used to manipulate, update, create, and disseminate data via SQL statements (Appendix 3). The system was effective in creating Database Management System (DBMS) tables, management of users, administration of the database, and derivation of SQL syntax. The command line on shp2pgsql-gui made it easy to import tables containing different shape files into the system.

\subsubsection{System implementation}

According to Agrawal and Gupta (2017) the framework of real-time geospatial data sharing has design components shown in Figure 3. The first element is the front end application, also referred as web's customer or client side. The customer or client side of the web usually points to the internet browser on the GIS software interface of the client's desktop or smartphone screen. In the web GIS application, it points out to where clients front end interface allows with dissemination of spatial data. This is where sending and receiving spatial requests or queries and issuing SQL statements such as select, get capabilities, and describe process are executed to the back end server occurs (Cao \& Wachowicz, 2019).

The second component is a server. that consists of an application server, database server, map server, and web site server. Basically these servers consist of stack of software that uses communications protocol (Hypertext Transfer Protocol) to serve consumers virtually with the documents that form Web pages in reaction to their demands that are transmitted by their computer communication protocol (Agrawal \& Gupta, 2017).

The application server is a component-based product that resides in the middle-tier of a server centric architecture (Agrawal \& Gupta, 2017; Wanjohi \& Mutua, 2015). In addition, according to (Magondu et al., 2012; Müller et al., 2016) application server provides middleware services for security and state maintenance, along with data access and persistence. It is a tailor made software that creates, convey and oversee substantial number of utilizations in a disseminated domain (Yang et al., 2015). The geodetic coordinate conversion system application development was put together via a sequence of tasks utilizing technologies such as application server, application cache, development of client end or front end, development of back end or server side of the application and finally website development (Lagmay et al., 2017). The coordinate system conversion framework was developed using python. 


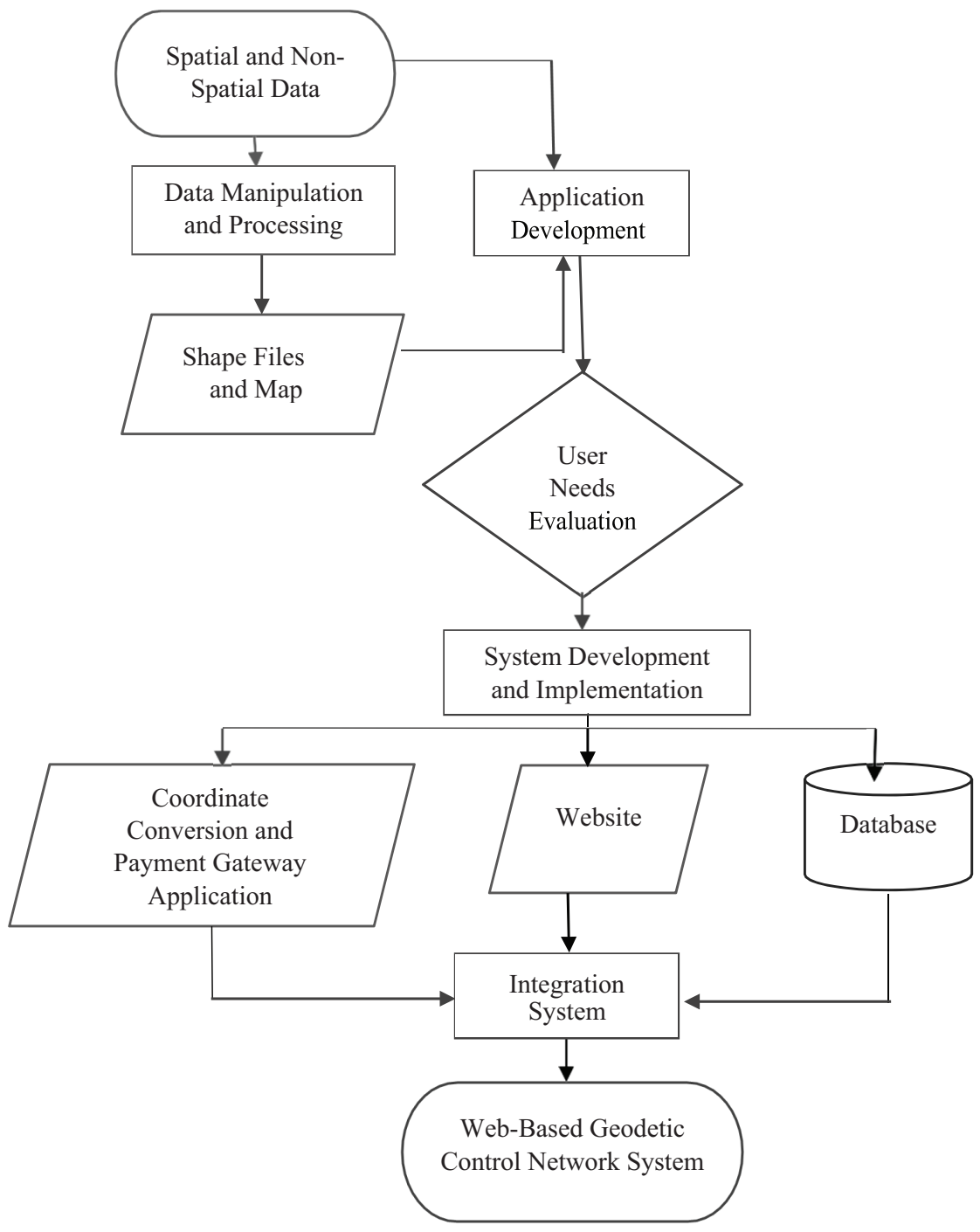

Figure 2. Approach adopted for the study

Front End (Client Side Web Browser)

Java Script, Apple Plugin, CSS

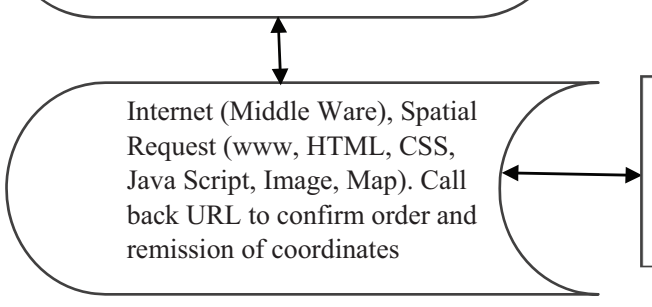

Application Back End Design

Web Server, API, Servlet, Python Django, PHP

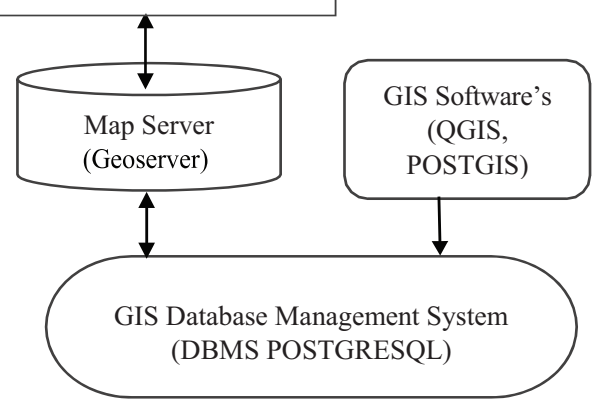

Figure 3. System implementation approach 


\subsubsection{Coordinate transformation}

The formula in Equations (i) and (ii) (2-D conformal transformations) were utilised for conversion of Cassini coordinates to UTM and vice versa (Ziggah et al., 2018). While the conversion formula used in transforming geographic coordinates from World Geodetic System 1984 (WGS84) (Harvey, 1986) ellipsoid to Universal Transverse Mercator (UTM) in Clarke 1880 ellipsoid and arc 1960 datum is Helmert transformation (Bursa wolf transformation model). The Bursa-Wolf (Bursa, 1962; Wolf, 1963) is a seven-parameter model for transforming three-dimensional Cartesian co-ordinates between two datums. This transformation model is more suitable for satellite datums on a global scale (Kutoglu et al., 2002). The transformation involves three geocentric datum shift parameters $(\Delta \mathrm{X}, \Delta \mathrm{Y}, \Delta \mathrm{Z})$, three rotation elements (RX, RY, RZ) and scale factor $(1+\Delta \mathrm{L})$ (Kutoglu et al., 2002). In the Conformal transformation, the equation is:

$$
\begin{aligned}
& X=s X \cos a)-s Y s)+x \\
& Y=s X \sin a)-s Y c)+Y .
\end{aligned}
$$

The simplified equation is:

$$
\begin{aligned}
& X=a X-b Y+x ; \\
& Y=b X+a Y+Y,
\end{aligned}
$$

where $a=s \cos a$ ) and $b=s \sin a)$.

The transformation parameters (or coefficients are $a$, $\left.b, x_{0}, y_{0}\right)$. The rotation is defined by one rotation angle $(a)$ and the scale change by one scale factor $(s)$. The translation is defined by two origin shift parameters $\left(x_{0}, y_{0}\right)$. In transformation from WGS84 ellipsoid to Clarke 1880 ellipsoid on arc 1960 the formulae used is as shown in Equation (v) below.

$$
\begin{aligned}
& \begin{array}{llllll}
X_{i} & 1 & \theta_{3} & -\theta_{2} & x_{i} & X_{0}
\end{array} \\
& {\left[Y_{i}\right]=\mu\left[\begin{array}{lll}
-\theta_{3} & 1 & \theta_{1}
\end{array}\right]\left[y_{i}\right]+\left[Y_{0}\right] ;} \\
& \begin{array}{llllll}
z_{i} & \theta_{z} & -\theta_{1} & 1 & z_{i} & Z_{0}
\end{array} \\
& \left.\begin{array}{ccccccc}
\Delta X_{i} & 100 & x_{i} & 0 & -z_{i} & y_{i} \\
{\left[\Delta Y_{i}\right]=} & 010 & y_{i} & z_{i} & 0 & -x_{i}
\end{array}\right]\left[\begin{array}{c}
\Delta X_{0} \\
\Delta Y_{0} \\
\Delta Z_{0} \\
\Delta \mu \\
\Delta Z_{i}
\end{array}\right.
\end{aligned}
$$

We embark by transforming to UTM from geographic coordinates equation (vii) is utilised. The plane UTM coordinates are calculated from the formulae given in equations, (vii, viii, ix and $\mathrm{x}$ ) the Easting measured from the central meridian positive eastwards A false origin of 500,000 metres is added to the Eastings.

$$
\begin{aligned}
& X(v+h) \cos \phi \cos \lambda \\
&(Y)=((v+h) \cos \phi \sin \lambda) ; \\
& Z \quad((v 1-e 2)+h) \sin \phi \quad(\text { vii }) \\
& S_{0}=A^{\prime}(\phi)-B^{\prime} \sin (2 \phi)+C^{\prime} \sin (4 \phi)-D^{\prime} \sin (6 \phi)+E^{\prime} \sin (8 \phi),
\end{aligned}
$$

where $\phi$ and all angles are expressed in radians.

The interactive viewer for displaying of spatial dataand client forms were coded using JavaScript and then linked to the website html and cascading style sheet (CSS). To ensure the retrieval of web maps and data was fast and efficient the web cache tiling technology was utilized via a Geoweb cache. Geoserver was utilized as map server. The payment gateway system was put together by combining the html and PHP scripts.

$$
\begin{aligned}
& \left.A^{\prime}=a\left[1-n+\left(\frac{5}{4}\right)\left(n^{2}-n^{3}\right)+\left(\frac{81}{64}\right) n^{4}-n^{5}\right) \ldots\right] \\
& B^{\prime}=\left[3 \tan \left(\frac{S}{2}\right)\right]\left(1-n+\left(\frac{7}{8}\right)\left(n^{2}-n^{3}\right)+\left(\frac{55}{64}\right)\left(n^{4}-n^{5}\right) \ldots\right) ; \\
& C^{\prime}=\left(15 \tan ^{2}\left(\frac{S}{48}\right)\left[1-n+\left(\frac{11}{16}\right) n^{2}-n^{3}\right) \ldots\right] ; \\
& D^{\prime}=35\left(\tan ^{3}\left(\frac{S}{48}\right)\left[1-n+\left(\frac{11}{16}\right) n^{2}-n^{3}\right) \ldots\right] ; \\
& E^{\prime}=315\left(\tan ^{4}\left(\frac{S}{512}\right)[1-n \ldots] ;\right. \\
& K_{3}=\frac{v \sin \phi \cos \phi}{24}\left(5-\tan ^{2} \phi+9 e^{2} \cos ^{2} \phi+4 e^{2} \cos ^{4} \phi\right) s f ; \\
& K_{2}=v \sin \phi \cos \phi\left(\frac{s f}{2}\right) ; \\
& K_{1}=S_{0} \times s f ;
\end{aligned}
$$$$
\text { Northing }=K_{1}+K_{2} p^{2}+K_{3} p^{4} \text {; }
$$

$K_{4}=s f(v) \cos (\phi)$

$$
\left.K_{5}=\left(\frac{s f v) \cos ^{3} \phi}{6}\right) \times 1-\tan ^{2} \phi+e^{2} \cos ^{2} \phi\right)
$$

Easting $=F E+K_{4} p+K_{5}$. 


\section{Results}

\subsection{User needs evaluation}

Results from user needs assessment survey indicates that obstacles that stands against the noble cause of design and implementation of the system for fast and easier data access include: manual and paper records, technology constraints, staff skills, and requirements for document authentication (Figure 4).

As per the assessment the system ought to have capability for web map visualisation, coordinates conversion, coordinates access via online payment, and dissemination of accessed geodetic data remotely via email (Figure 5).

\subsection{Geodetic control network geoportal}

The significant target of the research was to build up a geoportal for geodetic control network that will offer successful solution to remote data access. Results demonstrate that the geoportal implementation was fruitful as the coordinate's conversion, visualisation capability using different spatial catalogue layers, payment gateway, reception of coordinates remotely via email, database development and web servers were were tested (Appendix A).

Where according to (Gacoki \& Aduol, 2002), the used notations are described as follows; $v$ the radius of curvature of the ellipsoid in the prime vertical the radius of curvature of the ellipsoid in the meridian, $h$ ellipsoidal

Ministry of Lands Survey of Kenya Staff Response

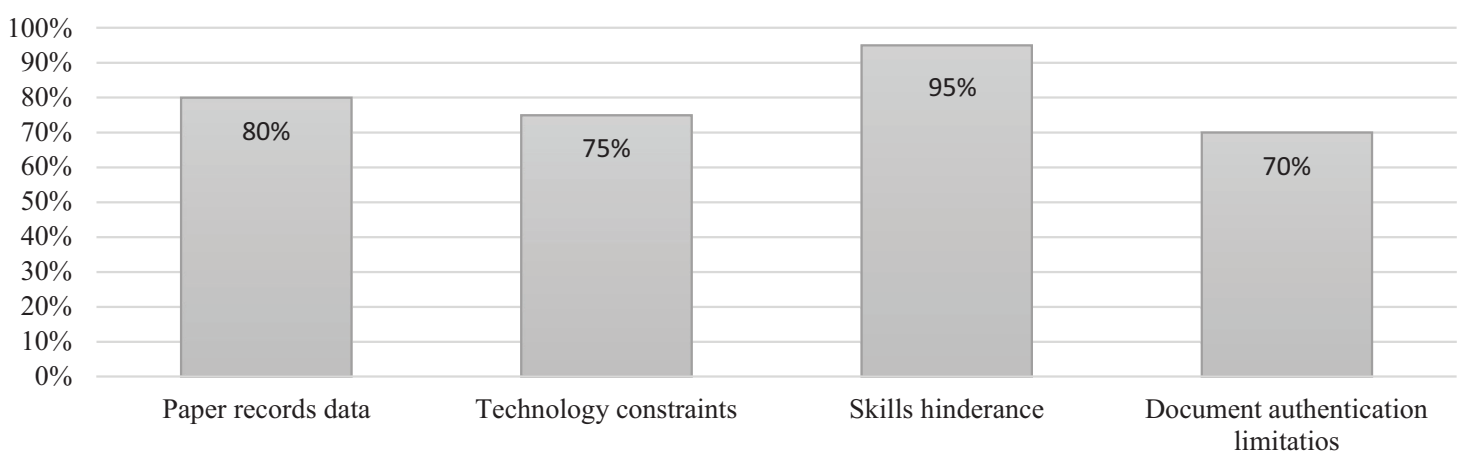

Figure 4. Staff response on system requirements
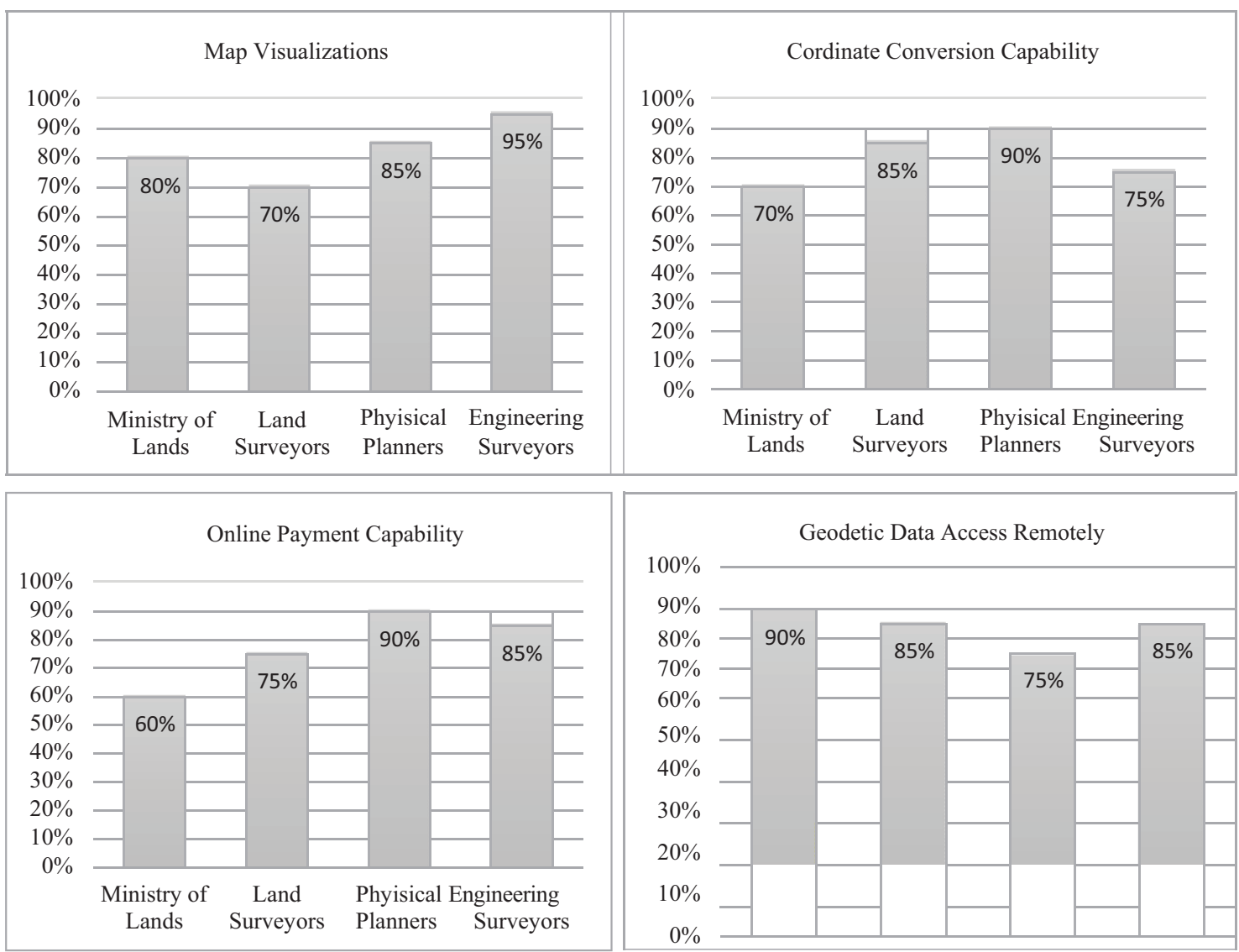

Figure 5. Response on working system functionalities 
height, $\phi$ The geodetic latitude, taken positive north of the equator, $\lambda$ The geodetic longitude measured from Greenwich positive eastwards, $\lambda_{0}$ The geodetic longitude of the central meridian $e$ eccentricity of the ellipsoid, $\lambda_{0}$. The meridian distance from the equator positive northwards, $f$ flattening of the ellipsoid, $K_{0}$ the UTM central meridian scale factor $=0.9996, a$ the major axis of the ellipsoid, $b$ the semi-minor axis of the ellipsoid, $\theta$ the parametric latitude, $\phi_{0}$. The geodetic latitude, taken positive north of the equator, $\phi \mathrm{p}$. The latitude for which $S_{0}=N / K_{0}, \phi \mathrm{p} 1$. The foot latitude, $N$. The Northings measured from the equator and taken positive northwards. In the southern hemisphere, a false origin of 10,000,000 metres is added to the Northings, $E$ effectively developed. All the functionalities of the system were tested (Appendix A).

\subsection{Web map visualizations}

The designed online visualisation platform is as shown in Figure 6. The platform was tested successful by a team of eight members from department of Geomatics and
Geospatial Information System (GEGIS) from in Jomo Kenyatta University of Agriculture and Technology.

The team navigated and interacted with the systems functionalities and confirmed that it was compatible with view and analysis tools as developed. These functionalities enable zoom, pan, identify point of interest, select and locate features of interest, refresh, print, about map, help, spatial catalogue layers overlay of street map, and layers overlay (satellite imagery, Africa layer, Kenya counties, towns, water bodies, lakes, rivers, Survey of Kenya pillars and destroyed pillars, photographs of trigonometric index cards and photograph) demonstrated as shown in (Figure 6) below.

\subsection{Coordinates system conversion}

The system as developed is capable of converting coordinates from UTM to Cassini Soldner, Cassini Soldner to UTM as well as from geographic to UTM. The coordinate conversion can be done to millimetre level root mean square error (Figure 7).

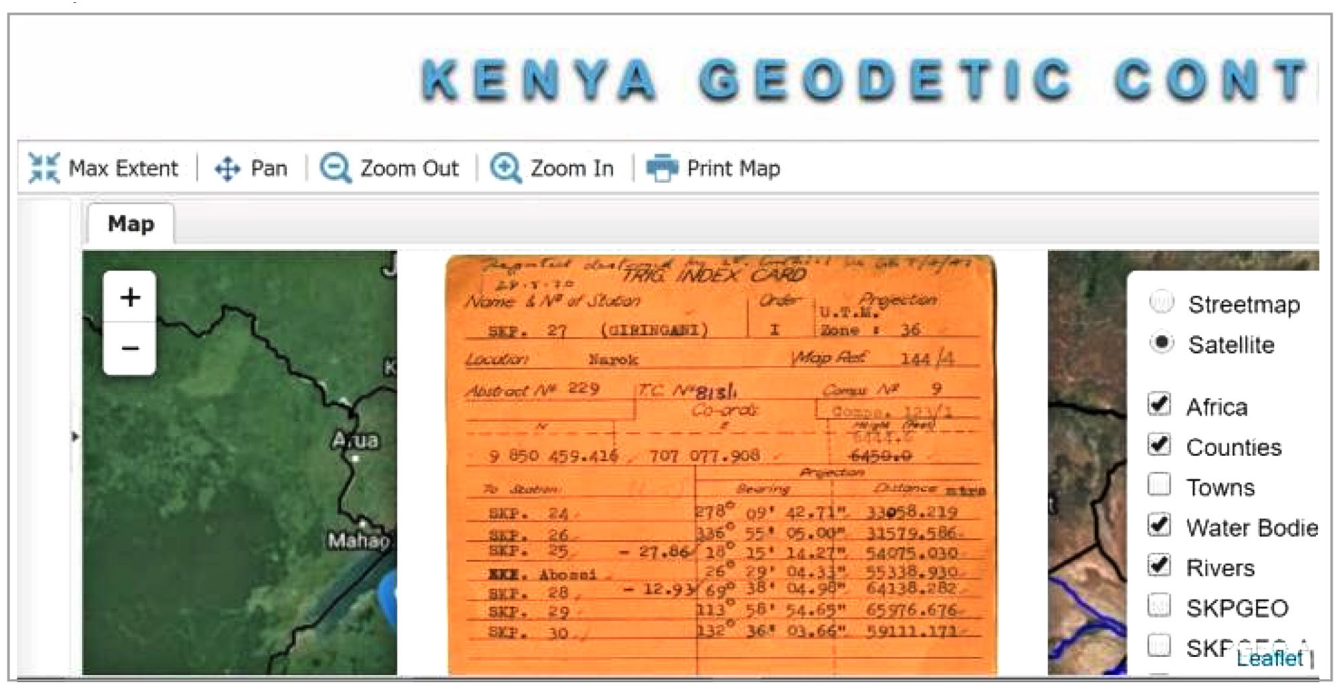

Figure 6. System application Interface with map visualization

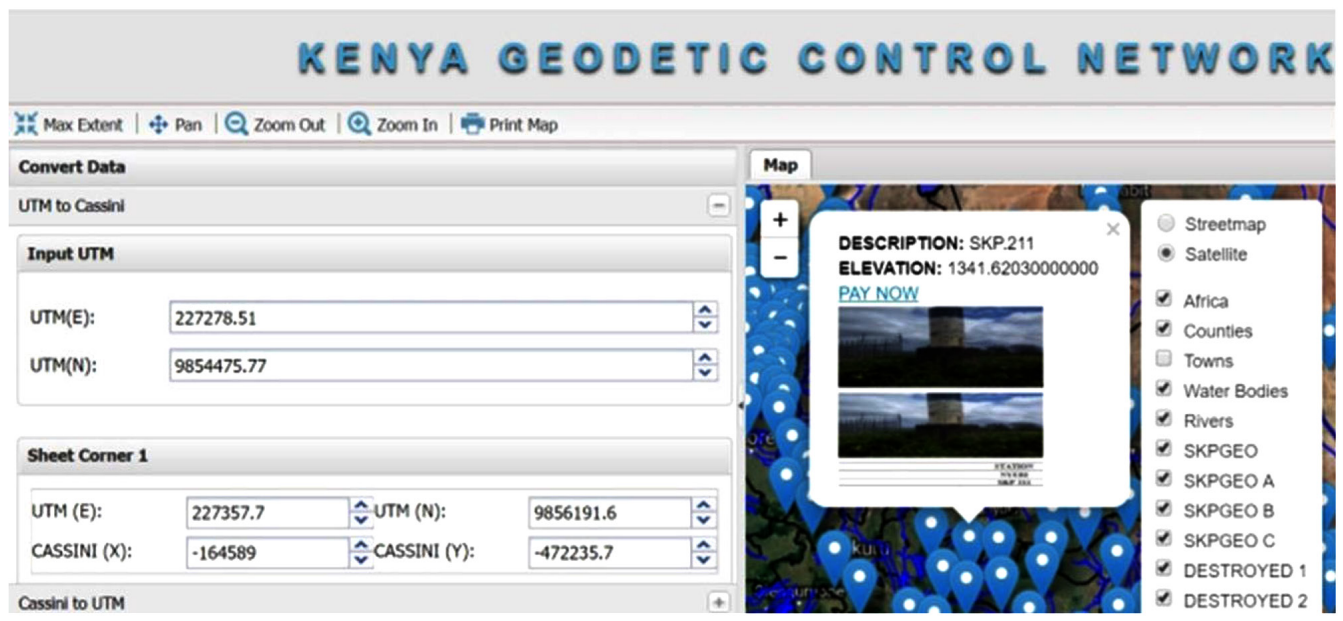

Figure 7. Coordinates conversion interface 


\subsection{Online coordinates payment gateway}

The application portal has a payment gateway (Figure 8) integrated into the mapping interface. The gateway capability was augmented in order for the system to disseminate the geographic coordinates of pillars remotely to the client directly from the database upon payment confirmation. The Figure 9 below shows the response from the system where to request for pillar information, the user enter required details and a payment of required system amount of $\$ 2$ hence the coordinated requested were automatically generated. Therefore, this shows that the user can access the coordinates at any time without any human input.

\section{Discussion}

The main aim of this research was to design a webGIS geoportal for management of geodetic control networks, dynamic visualizations and coordinate dissemination to users via a payment gateway. This webGIS platform eliminates inefficiencies related to the existing manual system which is associated errors in coordinate conversion $(\mathrm{Mu}-$ laku, 1997).

The web-based geoportal visualization platform has been found to make an immense contribution to professionals who need to identify Kenya pillars for use, in place of the manual topographic paper map (Basu, 2004). The implication of this is the inclusion of several layers of land use and land cover maps and imagery; that are overlaid in the system enhancing better visualisations that could not be achieved manually. Drawing inferences from the results obtained, the system is advantageous over other data sharing web portal. The web map services are not identified to be a component of the existing Land information system (LIMS) in the ministry. As a result, not much could be achieved in matters that refer to web visualization hence the adoption of the manual process. The payment of coordinates gateway was especially found to be helpful to raise the government revenues. The government get the revenues upon payment of ordered data and the client gets data remotely at any time. This saves cost for either party and eliminates congestion and job pile up at the ministry headquarters which is of great help in public health safety in the midst of the current COVID19 pandemic. The study has advanced in creation of spatial operations that other web GIS frameworks in the country have not made in roads to. The geodetic system framework in our study has a spatial overlay component. The user can visualise the location by incorporating different spatial layers to enhance decision making. Further the payment gateway, web processing and conversion of coordinates online is an additional feature that previous studies had not intervened (Figure 7 and 8).

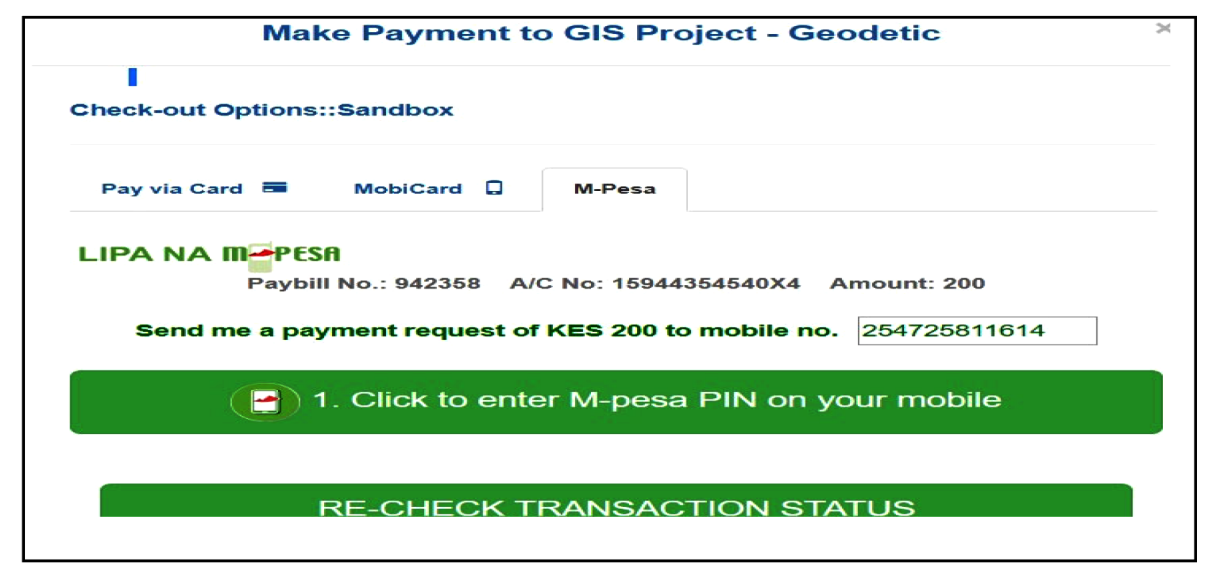

Figure 8. Payment Gateway

gisproject@mobi-remit.com via web5.us.freehostia.com
to me
Hello!
The coordinates you requested for transaction reference: 2960903 for 193. S2 are:
PILLAR:193.S2
DATUM: ARC 1960
NORTHING: 9640093.64
EASTING:625611.43
ELEVATION:1272.012
UTM ZONE: 37 SOUTH
COUNTY:KILIFI

Figure 9. Remission of coordinates remotely 
An online GIS system application was designed and implemented to tackle the gaps in access and use of geodetic information. The application has addressed the problem of manually identifying the location of the Kenya geodetic network pillars from topographic maps and geodetic data from trigonometric index cards. The paper datasets are centrally situated at the technical section in survey of Kenya field headquarters in Nairobi. For example, clients residing in all other counties in Kenya depend on this information; they have to travel to Nairobi, verify which pillar is close to their project region on a topographic hardcopy map, and choose pillars close to their points of interest.

If these pillars are demolished as is the case for some pillars, the customer has no means to restore them. Geodetic network control system can provide data bearing and coordinates to the other pillars so that one can restore the pillar at any location using either conventional survey methods such as using a total station or using global satellite navigation system. This portal can also tackle the difficulties of data accessibility; for instance, the data can be accessed automatically outside normally office working hours.

\section{Conclusion and Outlook}

Drawing inferences from the study, web-GIS application is a highly prospective advancement in GIS. Due to wide coverage of public accessing the web via internet, the web is the best medium for dissemination of data to the wider clients. This is advantageous because it eliminates services having to be centrally located somewhere in an office that is attributable to corrupt practices; bribes to officers facilitating the processing of paper record data. The government can also be denied from accessing the revenue, since there is no means of monitoring that the transactions occurred.

The research confirmed that there are three primary areas in the geodetic data application that are paramount; a system for conversion of co-ordinates online, request for access, payment of coordinates remotely and remitting geodetic data to the user online with these discrete components being resolved through the web based integrative strategy.

The research establishes the framework of legitimate administration of the land assets. It helps in acknowledgment of The Kenya Vision 2030. The research findings can be replicated to make land data Information systems framework that can give administration to a wide scope of clients, beginning with government establishments and private.

As can be confirmed from the study results, Web GIS applications abolish duplication and irregularity and facilitate spatial data access at a lower cost for each client intuitively and conveniently across organizations. The Kenya counties and national government through joint efforts should implement e-Government services so that the current paper land records can be authenticated, reorganized, updated to ensure coordination and information sharing between existing offices. This will facilitate prudent land administration, ensuring that technical data such as geodetic network control data that is pivotal for the decision makers and clients on land use and management is accessible. As a result, an effective framework for providing services to people is developed.

Kenya land information system has no spatial units and spatial representations. Integrating this platform with the current piecemeal land information system can support most of the procedures connected with land allocation and land title issuance, thereby eliminating troublesome corrupt cartels in land registries. A functional Land information system (LIMS) in Kenya can be designed and developed by adopting the findings in current research.

Henceforth scale it to include the cadaster comprising of people, (people and organizations); basic administrative units, security rights (encumbrances, mortgages, charges, easements), use rights (ownership, building rights, land tenure, freehold and leasehold) responsibilities, and restrictions (ownership rights); spatial units (parcels, and the legal space of buildings and utility networks); spatial sources (surveying), and spatial representations (geometry and topology).

Therefore, a research on future platform that can carry out web processing functionalities such as spatial analysis and geo-statistical analysis ought to be. The system needs scalability hence more research on additional processing capability needed.

\section{References}

Agrawal, S., \& Gupta, R. D. (2017). Web GIS and its architecture: a review. Arabian Journal of Geosciences, 10(23).

https://doi.org/10.1007/s12517-017-3296-2

Basu, S. (2004). E-government and developing countries: an overview. International Review of Law, Computers \& Technology, 18(1), 109-132.

https://doi.org/10.1080/13600860410001674779

Bruce, R. H. (1986). Transformation of 3D Co-ordinates. Australian Surveyor, 33(2), 105-125.

https://doi.org/10.1080/00050326.1986.10435216

Cao, H., \& Wachowicz, M. (2019). The design of an IoT-GIS platform for performing automated analytical tasks. Computers, Environment and Urban Systems, 74, 23-40.

https://doi.org/10.1016/j.compenvurbsys.2018.11.004

Gacoki, T. G., \& Aduol, F. W. O. (2002). Transformation between GPS coordinates and local plane UTM coordinates using the excel spreadsheet. Survey Review, 36(284), 449-462.

https://doi.org/10.1179/sre.2002.36.284.449

Kutoglu, H. S., Mekik, C., \& Akcin, H. (2002). A comparison of two well known models for 7-parameter transformation. Australian Surveyor, 47(1), 24-30. https://doi.org/10.1080/00050356.2002.10558839

Lwangasi, A. S. (1993). Datum transformation parameters for the Kenya geodetic system. Survey Review, 32(247), 39-46. https://doi.org/10.1179/sre.1993.32.247.39

Mabeya, M., \& Waithaka, H. (2014). Developing a geoportal for county SDI: case study of Nairobi County. International Journal of Science and Research, 3(9), 1965-1971. https://www.ijsr. net/search_index_results_paperid.php?id=SEP14510 
Magondu, M. G., Kuria, D. N., \& Ngigi, M. M. (2012). Webbased land information management system: a case study of nairobi city council. In Proceedings of the Annual Jomo Kenyatta University of Agriculture and Technology Scientific Conference (pp. 483-494). JUJA. http://journals.jkuat.ac.ke/index. php/jscp/article/viewFile/1077/881

Mugnier, C. (2014). Geodetic report of Kenya. ResearchGate, 1, 1-12. https://www.researchgate.net/publication/258509444_Geodetic_Report_of_Kenya

Mulaku, G. C. (1997). Land information management in Kenya. Civil Engineering, Research and Practice, 1(1), 75-88.

Müller, M. F., Thompson, S. E., \& Kelly, M. N. (2016). Bridging the information gap: A webGIS tool for rural electrification in data-scarce regions. Applied Energy, 171, 277-286. https://doi.org/10.1016/j.apenergy.2016.03.052

Murage, E. M., Gitimu, P., \& Sato, J. (2008). The project for strengthening survey of Kenya for GIS promotion in the Republic of Kenya. In The International Archives of the Photogrammetry, Remote Sensing and Spatial Information Sciences (Vol. XXXVII, Part B6a). Beijing. https://www.isprs.org/proceedings/XXXVII/congress/6a_pdf/3_WG-VI-3/06.pdf

Mwaura, D. W., \& Waithaka, H. (2013, January). Cloud GIS in geothermal resource data management: a case study of the Kenya Electricity Generating Company. In Spatial Enablement in Support of Economic Development and Poverty Reduction (pp. 277-298). GSDI Association Press. https://www. researchgate.net/publication/263133050

Lagmay, A. M. F. A., Racoma, B. A., Aracan, K. A., AlconisAyco, J., \& Saddi, I. L. (2017). Disseminating near-real-time hazards information and flood maps in the Philippines through Web-GIS. Journal of Environmental Sciences, 59, 13-23. https://doi.org/10.1016/j.jes.2017.03.014

Owino, B. N. (2013). Kenya country report to the 26th international cartographic conference to be held in Dresden Germany from 25th to 30th August (pp. 1-21). Republic of Kenya. https://icaci.org/files/documents/national_reports/2011-2015/ Kenya_2011-2013.pdf

Rainsford, H. F. (1951). The African ARC of the 30th Meridian. Empire Survey Review, 11(82), 159-163. https://doi.org/10.1179/sre.1951.11.82.159

Stone, J. C. (1976). Pioneer geodesy: the ARC of the 30th Meridian in the former Northern Rhodesia. The Cartographic Journal, 13(2), 122-128. https://doi.org/10.1179/000870476786961434

Wanjohi, S. G., \& Mutua, F. N. (2015). Developing a web based integrated land information system for ownership, value and taxation: case study of the Ministry of Lands, Housing and Urban Development, Kenya. International Journal of Science and Research (IJSR), 4(9), 984-991.

Wood, J. C. (2014). Sir David Gill and the Geodetics Survey of South Africa and the Arc of the 30th Meridian. Empire Survey Review, 7(48), 50-56. https://doi.org/10.1179/sre.1943.7.48.50

Yang, Y., Tang, J., Luo, H., \& Law, R. (2015). Hotel location evaluation: A combination of machine learning tools and web GIS. International Journal of Hospitality Management, 47, 14-24. https://doi.org/10.1016/j.ijhm.2015.02.008

Ziggah, Y. Y., Issaka, Y., \& Laari, P. B. (2018). 2D Cadastral Coordinate Transformation using extreme learning machine technique. Geodesy and Cartography, 67(2), 321-343. https://doi.org/10.24425/118706

\section{APPENDIX}

\section{Appendix 1. Geodetic Network Control System Link}

The developed geodetic network control system is highlighted in the web link below.

http://45.79.19.195, http://45.79.19.195/pay/SKP.211/. http://45.79.19.195:8080/geoserver/web/?1.

\section{Appendix 2. SQL Statement}

Question 1

Purpose: This query gives us detail of User and Ordered Item Details in month from current date

Query: select CONCAT(P. FirstName,P.LastName) as Name, P.MobileNo, I.ItemDesciption, I.Price from Person $\mathrm{P}$ join UserDetail U P.PersonID=U.PersonID join Order O U.UserID $=$ O.UserID join OrderedItems OI on O.OrderID=OI.OrderID join Item I on OI.ItemID=I. ItemID where DATE_PART('day', NOW()-O.OrderedDate) $<30$;
Question 2

Purpose: Cancel all those order whose Payment not paid till order requirement date and payment method is other than cash, because in online payment method order always place after payment.

\section{Query:}

Update Order O

set O.OrderStatus='Cancel'

where O.OrderID in (select O1.OrderID from Order O1 join Payment P on O.OrderID=P.OrderID where O1.OrderStatus != 'Complete' and PaidAmount $=0$ and Balance $>0$ and P.PaymentMethod !='Cash' and

DATE_PART('day', NOW()-O1.RequiredDate) $<0$ );

Question 3

Purpose: Give List of all member of selective department such as Geomatics and Geospatial Information System' 
Query: select CONCAT(P.FirstName,P.LastName) as Name, P.Email, P.MobileNo, P.Gender from Deptarment D join Member M on D.DeptID=M.DeptID join Person P on P.PersonID=M.PersonID where D.DeptName='Geomatics and Geospatial Information System;

Question 4

Purpose: Change user status with 'Not Eligible', if its ordered items cancel 10 or more time. by default user status is 'Eligible'

\section{Query:}

update UserDetail U

set U.UserStatus = 'Not Eligible'

where U.UserID in ( select U1.UserID from UserDetail U1 join Order $\mathrm{O}$ on U1.UserID $=$ O.UserID group by U.UserID having $\operatorname{count}\left({ }^{*}\right)>=10$ and O.OrderStatus='Cancel');

\section{Question 5}

Purpose: List all Stores Inventories and their items

Query: select S.StoreName, In.InventoryName, I.ItemDescription, I.ItemPrice, I.TotalQuantity from Store S join StoreLocation SL on S.StoreID=SL.StoreID join Inventory In on S.StoreID=In.InventoryID join Item I on In.InventoryID=I.InventoryID;

\section{Question 6}

Purpose: Place/ship order of 'VIP' Customer or User on day of order

\section{Query:}

Update Order O

set O.ShippedDate=CURRENT_DATE

where O.OrderID in ( select O1.OrderID from UserDetail U1 join Order O1 on U1.UserID=O1.UserID where DATE_PART('day', NOW()-O.OrderDate) $=0$ and U1.UserStatus='VIP');

\section{Question 7}

Purpose: List detail of Member/Staff Memeber whose salary is more than $1 \mathrm{k}$ within 1 year (from join date till today)
Query: select CONCAT(P.FirstName,P.LastName) as Name, P.Email, P.MobileNo, P.Gender, M.Salary from Person $\mathrm{P}$ join Member $\mathrm{M}$ on P.PersonID=M.PersonID join Department D on M.DepID = D.DeptID where Salary $>1000$ and DATE_PART('day', NOW()-

M.JoinDate) $<=365$;

Question 8

Purpose: List top 3 Member whose work most time in station (to manage station card and calculation)

Query: select M.MemberID, count(StationID) as NumberofTimes from Member $\mathrm{M}$ on P.PersonID=M. PersonID join MemberStation MS on M.MemberID=MS. MemberID join Station S on MS.StationID=S.StationID group by M.MemberID order by count(StationID) Limit 3 ;

Question 9

Purpose: List Detail of all Station

Query: select S.StationID, S.Location,

S.NorthCoordinates, S.EastCoordinates, S.Height, (select S1.StationName from Station S1 where S1.StationID=C. ToStationID) as StationName, C.Bearing, C.Distance from Station S join Coordinates C on S.StationID $=$ C.FromStation

Question 10

Purpose: List Detail of Pillars and their location detail

Query:select P.PillarID, P.PillarNumber, P.DestroyPillarNumber, L.Street, L.ZipCode, T.TownName, C.CityName, CO.CountryName from Pillars P join Location $\mathrm{L}$ on P.PillarLocationID=L.LocationID join Town $\mathrm{T}$ on L.TownID=T.TownID join City C on T.CityID=C.CityID join Country CO on C.CountryID=CO.CountryID 


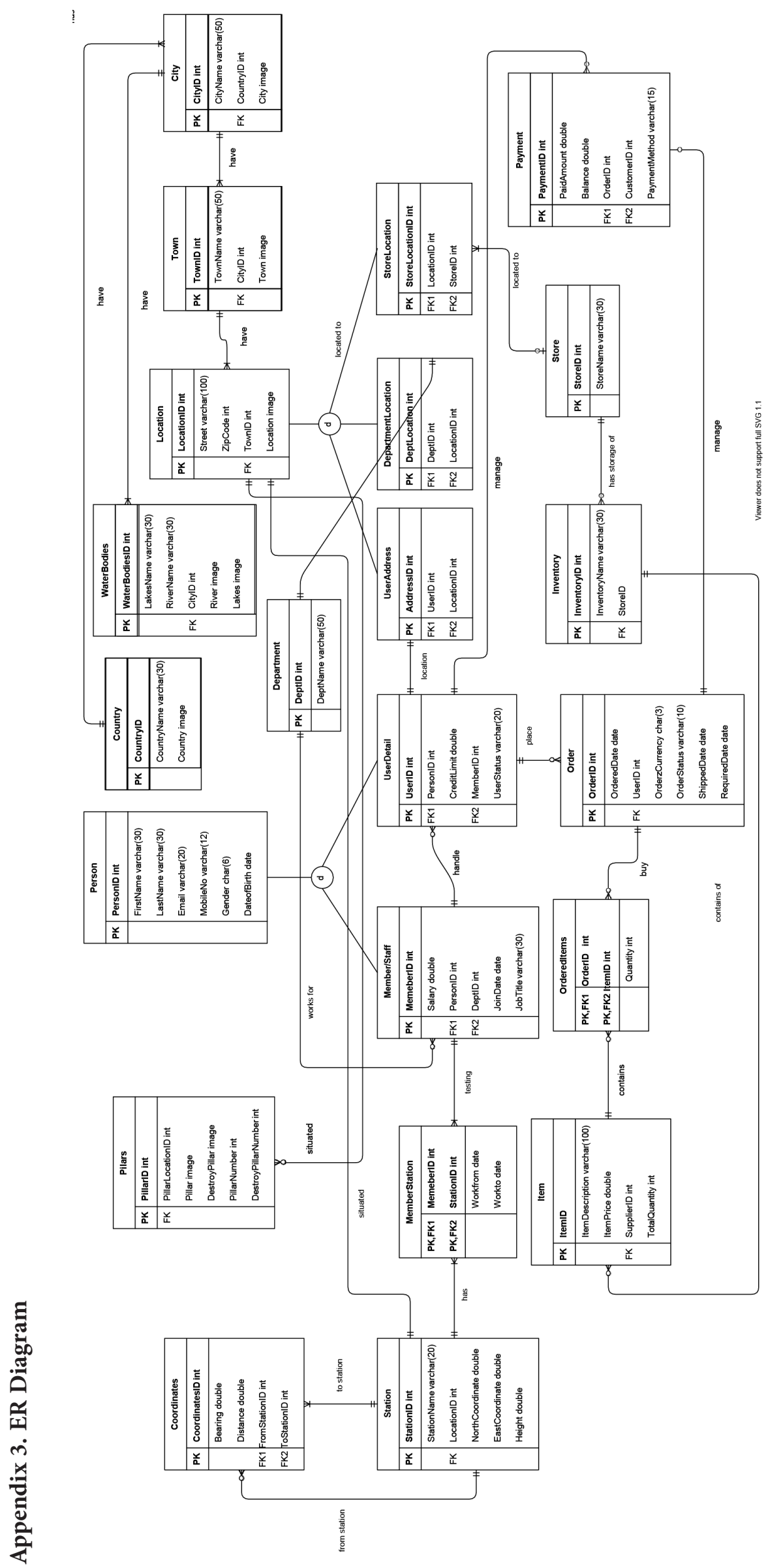

